\title{
EDITORIAL V. 7 N. 1 (2014)
}

ISSN: 1984-3151

\section{Magali Maria de Araújo Barroso}

\begin{abstract}
Doutora em Ciências em Engenharia de Sistemas e Computação. COPPE/UFRJ, 1987. Professora do Centro Universitário de Belo Horizonte - UniBH. Belo Horizonte, MG. magali.barroso@prof.unibh.br.
\end{abstract}

Nesta seção são apresentados dezoito artigos, realizados por pesquisadores brasileiros, que utilizam as Ciências Exatas e a Tecnologia como ferramentas em seu desenvolvimento. Atualmente a revista possui Qualis B4 nas áreas de Engenharia II, Ensino, Geografia e de Interdisciplinaridade, Qualis B5 em Ciências Sociais Aplicadas I, além de Qualis C em Ciências Ambientais, Ciência da Computação, Ciência de Alimentos e Química.

Os artigos selecionados, após a análise dos conselhos Científico e Editorial e de Pareceristas ad hoc, versam sobre temas: ANÁLISE ESPACIAL - GEOGRAFIA, CIÊNCIA dA ComputaçÃo - INTEligÊnCIA ARTificial, CiÊnCIA E Tecnologia de Alimentos, Ciências Ambientais e Biotecnologia, Educação - Engenharia QuímicA, EducAÇÃO MATEMÁtICA,, ENGENHARIA AMBIENTAL, ENGENHARIA DE PRODUÇÃO, ENGENHARIA DE TELECOMUNICAÇÕES, ENGENHARIA ELÉTRICA, ENGENHARIA QUÍMICA E, POR FIM, INTERDISCIPLINARIDADE: GEOGRAFIA, MATEMÁTICA E FísıCA.

A seguir encontram-se os resumos dos referidos artigos.

ANÁLISE ESPACIAL - GEOGRAFIA

O artigo Mapeando a Criminalidade Contra a Mulher No Município de Betim - MG: UMA Abordagem Exploratória realiza "uma análise espacial sobre a criminalidade contra a mulher no município de Betim sob a ótica da Lei Maria da Penha. A lei foi criada em 2006 com o propósito de prever assistência e proteção para a mulher em situação de violência. Utilizando a base de dados cedida pela Polícia Militar de Minas Gerais, foi possível fazer um roteiro metodológico nos anos de 2008 a 2010 relacionando tipos de crimes, bairros, quantidades de ocorrências e outros requisitos e analisar prováveis pontos que necessitam de campanhas educacionais em função do conhecimento das leis e suas sanções e policiamentos para minimizar esse transtorno, que é a violência doméstica. Os resultados dessa análise podem ser visualizados por meio de gráficos específicos e mapas da densidade espacial e os representativos dos crimes com maiores índices de registros." Os pesquisadores que realizaram a pesquisa foram Keila Pereira de Almeida e Bernardo Jeunon de Alencar, sendo, respectivamente, mestranda e doutor em Geografia - Tratamento da Informação Espacial da PUC Minas, MG. O último também é professor da mesma universidade.

CIÊNCIA DA COMPUTAÇÃO - INTELIGÊNCIA ARTIFICIAL

O "artigo apresenta a modelagem e implementação de um sistema de definição e classificação de níveis de assertividade para um Sprint de projeto utilizando-se a lógica fuzzy, com a finalidade de tornar mais eficiente o gerenciamento do desenvolvimento do software, 
auxiliando o analista de sistemas do projeto a ter uma tomada de decisão mais assertiva durante o processo do Sprint." Sistema de Classificação de Níveis de Assertividade de um Sprint de Projeto utilizandoSE Lógica Fuzzy é de autoria de Vinícius Nascimento Malheiros e Moisés Henrique Ramos Pereira, sendo o primeiro Bacharel em Ciência da Computação e o segundo mestre em Modelagem Matemática e Computacional pelo CEFET-MG e professor do UniBH.

\section{CiênCIA e TeCnOlogia de Alimentos}

"A análise sensorial pode ser utilizada para se evocar, medir, analisar e interpretar as reações dos consumidores em relação às características dos alimentos. Nesse contexto, os atributos olfativos são importantes para a escolha de um alimento. O objetivo deste trabalho foi de verificar a percepção sensorial das alunas do curso de pós-graduação na área de Nutrição, com uma abordagem didática aplicada pelo reconhecimento de aromas e aplicação de testes afetivos, como forma de aprendizado. Para a realização das análises, 30 alunas avaliaram 16 amostras (café, canela, cravo, vinagre, cachaça, azeite, orégano, molho shoyo, xarope de guaraná, açúcar, curry, pimenta calabresa, queijo parmesão, limão, vinho tinto e coentro). Também foram realizados os testes de preferência e intenção de compra de duas marcas de biscoitos amanteigados. Os resultados mostraram que os maiores índices de acertos foram verificados para os aromas das amostras de limão (84\%), café $(80 \%)$, canela $(80 \%)$, cravo $(80 \%)$ e vinagre $(80 \%)$; e que a pimenta calabresa e o coentro não foram reconhecidos por nenhum provador. Além disso, também foi observado que os biscoitos amanteigados da marca $A$ apresentaram maior aceitabilidade, quando $24 \%$ dos participantes afirmaram que certamente comprariam o produto. A partir dos resultados observados, foi possível verificar que as práticas realizadas podem contribuir para que o aluno exercite sua capacidade de perceber diferenças entre aromas de diferentes amostras de alimento e bebidas. Portanto, pode-se verificar a importância da análise sensorial como ciência, contribuindo de forma didática no aprendizado dos alunos do curso de Nutrição." Reconhecimento de aRomas e aPLicaÇÃo de testes afetivos CoMo FORMA DE APRENDIZADO tem a autoria de Kamila de Oliveira do Nascimento, Dilson Fagundes Ribeiro e Elga Batista, mestres da UFRRJ, RJ, sendo o segundo professor Instituto Federal de Educação, Ciência e Tecnologia do Espírito Santo - ES e a última do Centro Universitário Augusto Motta do Rio de Janeiro, RJ.

\section{CIÊnCIAs AmBIENTAIS E BIOTECNOLOGIA}

"As enzimas de origem microbiana apresentam características peculiares, que favorecem seu emprego em diversos processos biotecnológicos existentes, sendo relevantes as vantagens de conversões enzimáticas em diferentes processos industriais e ambientais. A sua produção, na maioria das vezes, ainda é economicamente dispendiosa, assim há necessidade de selecionar novos microrganismos com elevado potencial na produção de substâncias de alto valor agregado. A Caatinga é uma região pouco explorada biotecnologicamente, possuindo uma imensa população microbiana. Os rejeitos agroindustriais, principalmente os da indústria de alimentos, muitas vezes são descartados de maneira incorreta no meio ambiente e apresentam um elevado poder nutricional que poderia ser aproveitado na formulação de meios para produção de enzimas. A tanase é uma glicoproteína esterase formada predominantemente por um ácido gálico esterase e uma depsidase, além de possuir inúmeras aplicações biotecnológicas. Foram realizados estudos para seleção de 14 amostras de Aspergillus isoladas do solo da Caatinga de Pernambuco, para produção de tanase em meios alternativos contendo resíduos agroindustriais oriundos da indústria de sucos e bebidas (cascas de café, tangerina e uva). As 
fermentações submersas ocorreram a $30^{\circ} \mathrm{C}, 150 \mathrm{rpm}$, 120 horas. Os resultados obtidos indicaram que a amostra SIS 04 apresentou o maior halo de produção de tanase $(2,0 \mathrm{~cm})$ e uma atividade enzimática de 4,0 $\mu \mathrm{mol} / \mathrm{mim}$. $\mathrm{mL}$ de enzima, valor superior ao obtido no meio controle. A utilização de rejeitos agroindustriais para a elaboração de meios econômicos para a obtenção de bioprodutos de alto valor agregado surge como uma alternativa viável na produção de metabólicos com alto potencial biotecnológico." Este é o resumo do artigo UtILIZAÇÃo DE RESíduos AGROINDUSTRIAIS PARA PRODUÇÃO DE TANASE POR ASPERGILLUS SP ISOLADO DO SOLO DA CAATINGA DE PERNAMBUCO, BRASIL, desenvolvido por Katarina Botelho de Melo Nascimento, Alex Gabriel Rodrigues Martins, Galba Maria de Campos Takaki, Carlos Alberto Alves da Silva e Kaoru Okada da Universidade Católica de Pernambuco. A primeira autora é mestranda em Desenvolvimento de Processos Ambientais e o segundo faz iniciação científica em Educação Ambiental. Os três últimos são doutores, respectivamente, em Microbiologia, Biotecnologia e Medicina, pertencentes ao Núcleo de Pesquisas em Ciências Ambientais e Biotecnologia da UNICAP, PE.

"O setor de produção de enzimas microbianas tem crescido nas últimas décadas, motivado pela sua utilização nos diversos segmentos industriais. Por isso novas iniciativas em pesquisa resultam na produção de inovações dos processos e desempenho de produtos já existentes no mercado. Assim se observa que os fungos filamentosos compõem o grupo de microrganismos com maior número de espécies produtoras de compostos biotecnológicos, utilizados em diversos ramos industriais, e apresentam uma imensa variedade morfológica e um elevado potencial bioquímico e fisiológico. Nesse aspecto o gênero Aspergillus se destaca por apresentar uma grande capacidade na produção de bioprodutos de alto valor agregado, principalmente em enzimas microbianas. A seleção adequada dos nutrientes na elaboração dos meios de produção de lipase envolve uma série de estudos prévios para introdução de substratos que quimicamente sejam constituídos de ácidos graxos de cadeia longa e possam produzir a enzima em maiores quantidades. A Caatinga é uma região pouco explorada biotecnologicamente, possuindo uma imensa população microbiana, que apresenta um elevado potencial enzimático desconhecido. Foram realizados ensaios de seleção utilizando 6 meios de produção diferentes para produção de lipase, usando as amostras, denominadas SIS 10 e SIS 16, isoladas da Caatinga de Pernambuco. Os ensaios de produção ocorreram a $150 \mathrm{rpm}, 28^{\circ} \mathrm{C}$, durante 144 horas. Os resultados obtidos evidenciaram que o melhor meio testado foi o meio 4 , com a amostra SIS 16 , obtendo uma produção de $2,16 \mathrm{U} / \mathrm{mg}$ de lipase. $O$ estudo da seleção de meios de produção de enzimas microbianas é um recurso necessário para obtenção de novos meios com elevado potencial de produção." Este artigo intitulado SELEÇÃo de MEIOS DE PRODUÇÃo DE LIPASE POR AMOSTRAS DE ASPERGILLUS SP ISOLADAS da caatinga de Pernambuco foi desenvolvido por: Brindize Ferreira de Lima, Henrique Siqueira Amorim, Aline Elesbão do Nascimento, Galba Maria de Campos Takaki e Carlos Alberto Alves da Silva. A primeira autora é mestranda em Desenvolvimento de Processos Ambientais, o segundo é bacharelando em Engenharia Química, ambos da UNICAP, PE. Os demais autores são doutores, respectivamente, em Ciências Biológicas, Microbiologia e Biotecnologia, professores e membros do Núcleo de Pesquisas em Ciências Ambientais e Biotecnologia da UNICAP, PE.

EDUCAÇÃo - ENGENHARIA QUÍMICA

"Ao se observar as barreiras encontradas por acadêmicos de períodos distintos durante a graduação em Engenharia Química da Universidade Tecnológica Federal do Paraná - Campus Ponta Grossa (UTFPR $P G)$, constatou-se que as principais dificuldades envolviam o correlacionamento das matérias básicas da área de Engenharia e o reconhecimento acerca da 
importância do aprendizado envolvido nessas disciplinas. Inserido na função de auxiliar na formação interdisciplinar do acadêmico a fim de buscar soluções para as problemáticas em questão, o Programa de Educação Tutorial do curso de Engenharia Química (PET EQ) da UTFPR - PG, caracterizado por ações implementadas junto às graduações das Instituições de Ensino Superior, desenvolveu a Atividade Integralizadora. A atividade buscou facilitar o processo de aprendizagem do acadêmico e permitir a ele a visualização das áreas de atuação do profissional Engenheiro. A realização de uma abordagem lúdica e dinâmica instigou os acadêmicos a provar o conhecimento adquirido nas salas de aula e provocou abertura a novos conceitos de ensino." $O$ artigo intitulado Atividade INTEgralizadora: O ENSINO DE ENGENHARIA SOB UMA NOVA PERSPECTIVA é de autoria de Bruno Edward Meyer, Denilton da Conceição Fritz Junior, Elis Regina Duarte, Maria Cecilia Meneguette Ferreira e Paula Cristiana de Freitas, graduandos e doutora em Engenharia Química do UTFPR-PG, PR.

\section{EdUCAÇÃo MATEMÁTICA}

[...] "estuda a influência da linguagem simbólica na matemática. [...] A maioria dos estudos e pesquisas realizadas na área de educação matemática parte do pressuposto de que a matemática participa da formação dos indivíduos e é importante na sua inserção social. Neste sentido um insucesso poderia significar não apenas um fracasso na vida escolar, mas também na própria condição de cidadão dos indivíduos. Um dos problemas que pode dificultar a compreensão matemática de alguns estudantes é a dificuldade que eles têm de utilizar a simbologia necessária para o aprendizado dessa disciplina." A LINGUAGEM MATEMÁTICA NA SALA DE AULA: PERSPECTIVAS E DIFICULDADES tem como autor Roberto Lírio Florenço Junior, que é pós-graduado em Matemática e é professor da rede Estadual de Escolas Públicas de Santa Luzia, MG.
ENGENHARIA AMBIENTAL

"A água é o maior recurso natural e de grande importância para a manutenção da vida de todas as espécies do planeta. Esse recurso natural cobre cerca de $70 \%$ da superfície terrestre, entretanto cerca de 3\% deste volume é de água doce, que é o foco" do artigo Protocolo para biorremediação de águas CONTAMINADAS POR PETRÓLEO E DERIVADOS, de autoria de Ana Mara Araújo, Cinthia Gonçalves, Eduarda Moreira Nascimento, Juarez Moreira Júnior, Julyane Carolina Silva, Magno André de Oliveira, Patrícia Brisa, Pedro Henrique Pires e Elayne Cristina Machado. A última autora é Mestre em Ciências dos Alimentos pela UFMG e Professora do Centro Universitário de Belo Horizonte - UniBH. Os demais são graduandos de Engenharia Ambiental da mesma Instituição.

"A preocupação com a possível escassez dos recursos naturais é a principal justificativa para o aumento da busca pela sustentabilidade. Desta forma, a cada dia, procuram-se materiais e técnicas que minimizem os impactos ambientais. O artigo TIJOLO DE SOLO-CIMENTO: ANÁLISE DAS CARACTERÍSTICAS FÍSICAS E VIABILIDADE ECONÔMICA DE TÉCNICAS CONSTRUTIVAS SUSTENTÁVEIS tem por objetivo mostrar, através do tijolo-solo cimento, técnicas de construções sustentáveis", comprovando [...] "sua melhor resistência e durabilidade, por meio de testes e análises, comparando-o ao tijolo tradicional." A autoria é de Jessica Campos Soares Silva Motta, Paola Waleska Pereira Morais, Glayce Nayara Rocha, Joicimara da Costa Tavares, Gabrielle Cristina Gonçalves, Marcela Aleixo Chagas, Jalson Luiz Mageste, alunos de Engenharia Civil do UniBH e Taiza de Pinho Barroso Lucas, mestre em Geografia pela UFMG e professora do UniBH.

\section{ENGENHARIA DE PRODUÇÃO}

"A aplicação das ferramentas integrantes da abordagem Seis Sigma tem sido frequentemente 
utilizada pelas empresas. O presente artigo apresenta aplicação do método DMAIC (Define, Measure, Analyse, Improve, Control) para reduzir paradas de produção por falta de insumos de embalagem em uma indústria de computadores. Os atrasos na produção possuem impacto direto no resultado financeiro da empresa, principalmente em razão do atraso no faturamento das ordens de produção. A utilização de ferramentas aplicadas ao DMAIC possibilitou a coleta e análise dos dados para avaliar o problema. $O$ cenário projetado comparado com o atual estima redução de 48,24\% do valor monetário dos pedidos parados e 4\% no custo de aquisição dos insumos para o processo." O artigo APLICAÇÃo do MÉTOdo DMAIC PARA ANÁLISE DE PROBLEMAS DE PRODUÇÃO: UM ESTUDO DE CASo tem como autores Bruno Alves Almeida Braitt e Diego de Castro Fettermann, respectivamente, bacharel em Engenharia de Produção pela UESC BA e Doutor em Engenharia de Produção pela UFRGS - RS e professor da UESC - BA.

"A importância do gerenciamento de estoques é decorrente da busca por um melhor nível de atendimento ao cliente, fator este decisivo para a competitividade das empresas. Esse trabalho tem por objetivo a aplicação de um método para gerenciamento do estoque de produtos acabados a partir do nível de serviço em uma pequena empresa do setor eletrônico. Como principal resultado é apresentado o desenvolvimento do método realizado, a partir de um diagrama de causa e efeito dos problemas da empresa e a aplicação das nove etapas do método proposto. A aplicação do método proposto foi considerada de fácil assimilação na empresa e seus resultados permitiram sistematizar o processo de tomada de decisão quanto ao gerenciamento do estoque e minimizar os problemas de não atendimento dos pedidos por falta de produtos." O artigo intitulado Proposta para Gestão de Estoques em Uma EMPRESA DE BASE TECNOLÓGICA é de autoria de Graziele Bueno Hugo, Diego de Castro Fettermann e
Giovana Savitri Pasa, sendo a primeira autora bacharel e os demais doutores em Engenharia de Produção pela UFRGS - RS. A primeira é Engenheira de Produção da AMBEV - RS, o segundo, professor da UESC - BA e, a terceira, é professora da UFRGS RS.

\section{ENGENHARIA DE TELECOMUNICAÇÕES}

O artigo intitulado AplicaçÃo e Avaliação dE Modelos para Predição Celular na Área Urbana DE Conselheiro LAFAiEte "apresenta um estudo de predição celular na área urbana da cidade de Conselheiro Lafaiete. O objetivo é realizar o estudo de predição de sinais de radiofrequência, utilizando métodos computacionais para estimar níveis dos sinais e realizar medições em campo a fim de analisar e comparar os resultados. Primeiramente foi escolhida uma BTS (Estação Transceptora Base) na cidade de Conselheiro Lafaiete. Níveis de sinal foram obtidos na região de cobertura da referida estação e através dos parâmetros de transmissão da estação foram obtidos valores de recepção em diversos pontos da região de estudo, utilizando modelos de propagação implementados computacionalmente. Os níveis de sinal obtidos através das simulações foram comparados com as medições realizadas na área de cobertura da BTS e os resultados são apresentados a fim de mostrar a aproximação entre os modelos semiempíricos e as medições realizadas em campo. Tem como autores Ygor Bruno Fernandes da Silva e Paulo Tibúrcio Pereira, sendo o primeiro Bacharel em Engenharia de Telecomunicações pela UFSJ e o segundo doutor em Engenharia pela PUC Minas e professor da UFSJ - Ouro Branco, MG.

\section{ENGENHARIA ELÉTRICA}

"O crescente aumento na demanda do sistema elétrico, aliado à escassez de recursos de geração, tem aumentado a busca de novas tecnologias para melhorar o aproveitamento dos recursos existentes com a constante preocupação de se minimizar os 
investimentos. O artigo O Fator de PotênCIA EM Unidades Consumidoras Residencials objetiva analisar os valores de potência reativa em residências e diminuir esses valores para que melhore ainda mais a qualidade energia fornecida no país". Sua autoria é de Frederico Santiago Romualdo Rios e Gustavo Sacchetto Curi, engenheiros eletricistas formados pelo UniBH e projetistas das empresas Eletromecap Industrial Ltda e Projetos em Engenharia Ltda, respectivamente em Belo Horizonte, MG. Os outros autores: Fabricio Silveira Chaves, doutor em Engenharia elétrica pela UFMG e professor do UniBH e Arlete Vieira da Silva, mestre em Geografia e Análise Ambiental pela UFMG e consultora da S \& V Consultoria e Serviços Ltda, Belo Horizonte, MG.

ENGENHARIA QUÍMICA

O artigo intitulado Avaliação do TemPo dE Degradação do Coagulante natural Moringa oleifera lam em pó no tratamento de Água SUPERFICIAL "propõe o estudo do tempo de degradação do coagulante $M$. oleifera em pó, a fim de verificar se o poder coagulante é alterado em relação ao tempo de armazenamento desse produto em refrigerador. Os ensaios de coagulação/floculação e sedimentação foram realizados em Jar Test, com água bruta coletada na Sanepar, proveniente da Bacia do Rio Pirapó, Maringá, Paraná, com turbidez inicial de 80 NTU. Utilizou-se a dosagem de $50 \mathrm{mg} \cdot \mathrm{L}^{-1}$ do coagulante. Para a avaliação do tempo de degradação, analisou-se a redução percentual de cor aparente, turbidez e compostos com absorção em $U_{254 n m}$. Verificou-se que é interessante utilizar o coagulante durante o período de até 1 semana, pois as propriedades coagulantes tendem a diminuir com o tempo de armazenamento. Pode-se considerar que o pó das sementes de $\underline{M}$. oleifera destaca-se como um produto sustentável bastante atrativo para a purificação da água, sendo uma alternativa ambientalmente correta". De autoria de Karina Cardoso Valverde, Priscila Ferri Coldebella, Letícia
Nishi, Grasiele Scaramal Madrona, Franciele Pereira Camacho, Tássia Rhuna Tonial dos Santos, Onélia Aparecida Andreo dos Santos e Rosângela Bergamasco, sendo todas elas doutorandas, doutoras, pós-doutorandas, pós-doutoras e professoras de Engenharia Química pela UEM, PR.

Busca [...] "avaliar a utilização do ácido sulfúrico $\left(\mathrm{H}_{2} \mathrm{SO}_{4}\right)$ como catalisador na reação de esterificação dos ácidos graxos livres (AGLs) do óleo da polpa de Macaúba utilizando metanol e etanol. Os experimentos avaliaram o efeito do percentual de catalisador, razão molar AGLs:álcool, temperatura e tempo de reação. Os resultados obtidos mostram que o metanol é mais eficiente na esterificação dos AGLs, quando comparado ao etanol. Verificou-se que o aumento do percentual de catalisador influenciou significativamente no aumento da conversão da reação, para ambos os alcoóis. Avaliou-se o efeito da razão molar (AGL:álcool) de 1:1; 1:6; 1:9; 1:12; $1: 15$ e $1: 18$, sendo verificadas para o etanol conversões de $\sim 61 \%$ sob a razão molar $1: 9$, e conversões na ordem de $93 \%$ foram obtidas sob razão molar 1:18 para o metanol. Em razão molar AGLs: metanol 1:18, 2\% de catalisador e 60 minutos de reação, verificou-se que o aumento da temperatura reacional promoveu aumento na conversão dos AGLs na faixa de $30 \stackrel{\circ}{\circ}$ a $50 \stackrel{\circ}{\circ}$." O artigo ESTERIFICAÇÃO HOMOGÊNEA DOS ÁCIDOS GRAXOS LiVReS do Óleo da POLPA de MACAúba (ACrocomia ACULEATA) é o resultado da pesquisa de um grupo da UEM e Universidade Paranaense, ambas de Umuarama, PR, formado por Djéssica Tatiane Rasp, Bruna Tais Ferreira de Mello, Pablo Jorge da Silva e Camila da Silva. A primeira autora é mestranda em Bioenergia, dois são graduandos em Química Industrial e a quarta é doutora e professora da UEM.

[...] O "trabalho realiza um estudo de caso para avaliar as causas de uma baixa campanha de um tijolo refratário usado no distribuidor de uma usina siderúrgica, através da análise Post-Mortem. Foram coletados dois tijolos da região afetada, e ainda a 
escória que interagiu com tais tijolos foi analisada. Foram realizados testes de análise química por fluorescência de raios- $x$, difração de raios- $x$, perda ao fogo, microscopia, densidade e porosidade. De acordo com a análise Post-Mortem, comprovou-se que os tijolos do distribuidor sofreram infiltrações de aço e escória. Ao analisar esta última, percebeu-se tratar-se de uma escória ácida, com MgO de saturação superior ao teor livre. Além disso, sua temperatura de líquidus indicou uma escória fluida, ainda mais agressiva ao refratário. Por esse motivo, a massa de cobertura do distribuidor, utilizada como revestimento de trabalho, não suportou as condições de operação, e o distribuidor passou a operar sob o revestimento permanente, que não foi desenvolvido para trabalhar diretamente com o metal líquido.” AvaliAçÃo DE REVESTIMENTO REFRATÁRIO NO PROCESSO SIDERÚRGICO é de autoria de Luiza Santos Cotta e Christianne Garcia Rodrigues, sendo a primeira autora Bacharel em Engenharia Química e, a segunda, mestre em Engenharia Metalúrgica pela UFMG e professora do UniBH.

[...] este artigo tem "como objetivos analisar a composição química de óleos essenciais extraídos de folhas frescas de tomilho, capim-limão, alfavaca e manjericão, suas atividades antioxidantes pelas metodologias do sequestro dos radicais $\mathrm{DPPH}$ e da inibição da oxidação do sistema do $\beta$-caroteno/ácido linoleico." São "avaliados os potenciais antioxidantes dos padrões dos constituintes majoritários dos referidos óleos essenciais timol, citral, eugenol e linalol, respectivamente, usando-se como controles positivos os antioxidantes sintéticos ácido ascórbico, BHA e BHT." Adicionalmente, objetiva-se "correlacionar as composições químicas e concentração de $50 \%$ de inibição $\left(\mathrm{Cl}_{50}\right)$ dos referidos óleos essenciais por meio da análise de agrupamentos hierárquicos (HCA). Os constituintes majoritários presentes nos óleos essenciais das folhas frescas de tomilho, capim-limão, alfavaca e manjericão são o timol, p-cimeno e $\mathrm{y}$-terpineno; o geranial, neral e mirceno; eugenol, ( $\mathrm{Y}$ )-muuruleno e (cis)- $\beta$-ocimeno; cineol, linalol e cânfora, respectivamente. As amostras com atividades antioxidantes mais expressivas são o eugenol e o óleo essencial de alfavaca. Utilizando-se a HCA, observa-se que os óleos essenciais e seus respectivos padrões se agrupam devido à similaridade quanto às quantidades de fenilpropanoides $e$ monoterpenos. Este estudo pode ser importante para encontrar e analisar novos compostos de origem natural que permitam inibir a oxidação." CoRRELAção ENTRE COMPOSIÇÃO QUÍMICA E EFICÁCIA ANTIOXIDANTE DE ÓlEOS ESSENCIAIS DE PLANTAS CONDIMENTARES POR AnÁlise de Agrupamentos Hierárquicos (HCA) tem como autores Cíntia Alvarenga Santos Fraga de Miranda, Maria das Graças Cardoso, Samísia Maria Fernandes Machado, Marcos de Sousa Gomes, Juliana de Andrade e Maria Luisa Teixeira, um grupo de pesquisadores doutoras e doutorandos, a maioria em Ciência em Agroquímica da UFLA, MG.

INTERDISCIPLINARIDADE: Geografia, MatemÁticA E FísıCA

"Propõe-se refazer a medida da circunferência da Terra como uma estratégia similar à empregada por Eratóstenes na Antiguidade, porém utilizando as comunidades de jogos on-line e as redes sociais da internet. Foram envolvidas diferentes disciplinas, tais como Física, Matemática, Geografia e História para buscar a interdisciplinaridade. Alunos localizados em duas cidades distintas mediram a sombra de varetas com comprimento conhecido, no mesmo dia e hora. Tais cidades, que possuem longitudes aproximadas, mas, latitudes bastante distintas, foram localizadas com a ajuda do software Google Earth. Efetuadas as medições, calculou-se a circunferência meridional da Terra. Os resultados para o ensino das disciplinas envolvidas foram uma maior participação dos alunos e a oportunidade de compreenderem as correlações entre as diversas áreas do conhecimento envolvidas no projeto, situando a proposta no âmbito da 
pedagogia de projetos." A autoria do artigo MEdindo o Planeta Terra: Um Experimento com Abordagem INTERDISCIPLINAR é de Juliana de Fátima Silva e Samuel Bueno Soltau. Sendo a primeira autora Licenciada em Geografia pela UNIFAL-MG e o segundo doutor em Educação pela UERJ e professor da UNIFAL-MG.

O Quadro 1 mostra os artigos distribuídos conforme a natureza e instituições, cidades e estados da federação. Pode-se verificar que menos de $28 \%$ dos artigos são do UniBH, instituição responsável pelo periódico.

O Gráfico 1 mostra o número de artigos conforme a natureza das instituições e o Gráfico 2 indica o número de artigos por estados brasileiros. Pode-se notas que esta edição da revista contém artigos das regiões nordeste (3), sudeste (11) e sul (4).

Quadro 1 - Número de artigos por Instituição, Cidade e Estado.

\begin{tabular}{|c|c|c|c|c|}
\hline $\begin{array}{l}\text { Natureza da } \\
\text { Instituição }\end{array}$ & Instituição & Cidade & Estado & Número de artigos \\
\hline Particular & UniBH & Belo Horizonte & $M G$ & 5 \\
\hline \multirow{2}{*}{ Confessional } & PUC Minas & Betim & MG & 1 \\
\hline & INICAP & Recife & PE & 2 \\
\hline \multirow{4}{*}{ Estadual } & REEP & Santa Luzia & MG & 1 \\
\hline & UESC & Ilhéus & BA & 1 \\
\hline & \multirow{2}{*}{ UEM } & Maringá & \multirow{2}{*}{ PR } & 1 \\
\hline & & Umuarama & & 1 \\
\hline \multirow{6}{*}{ Federal } & UFAL & Alfenas & \multirow{3}{*}{ MG } & 1 \\
\hline & UFLA & Lavras & & 1 \\
\hline & UFSJ & Ouro Branco & & 1 \\
\hline & IFTES & Vitória & ES & 1 \\
\hline & UFRGS & Porto Alegre & RS & 1 \\
\hline & UTFPR & Ponta Grossa & PR & 1 \\
\hline
\end{tabular}

Gráfico 1

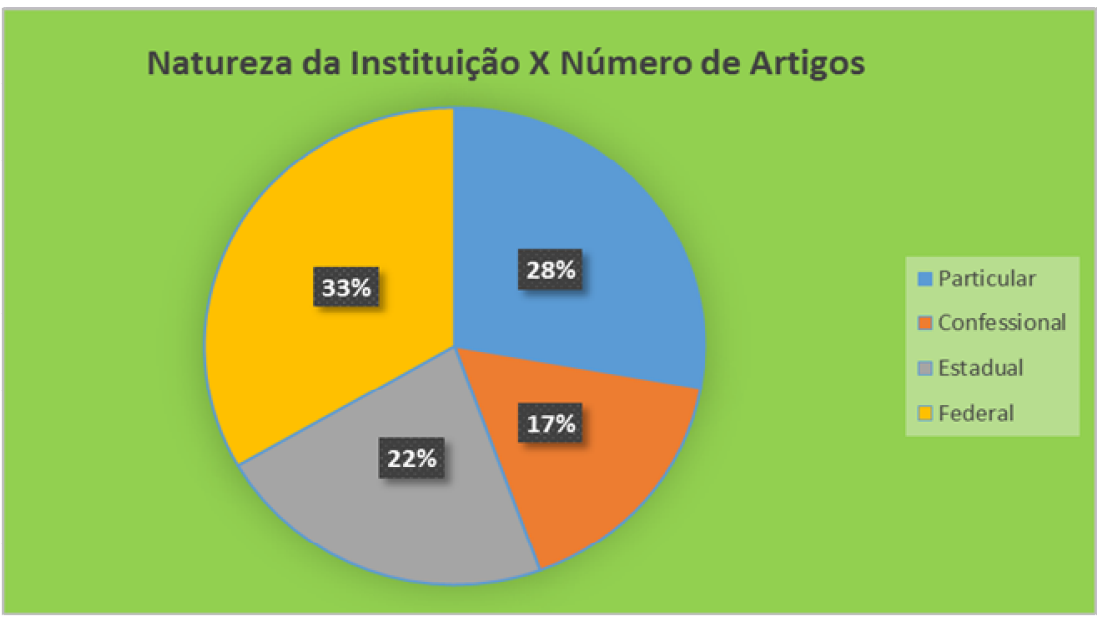




\section{Gráfico 2}

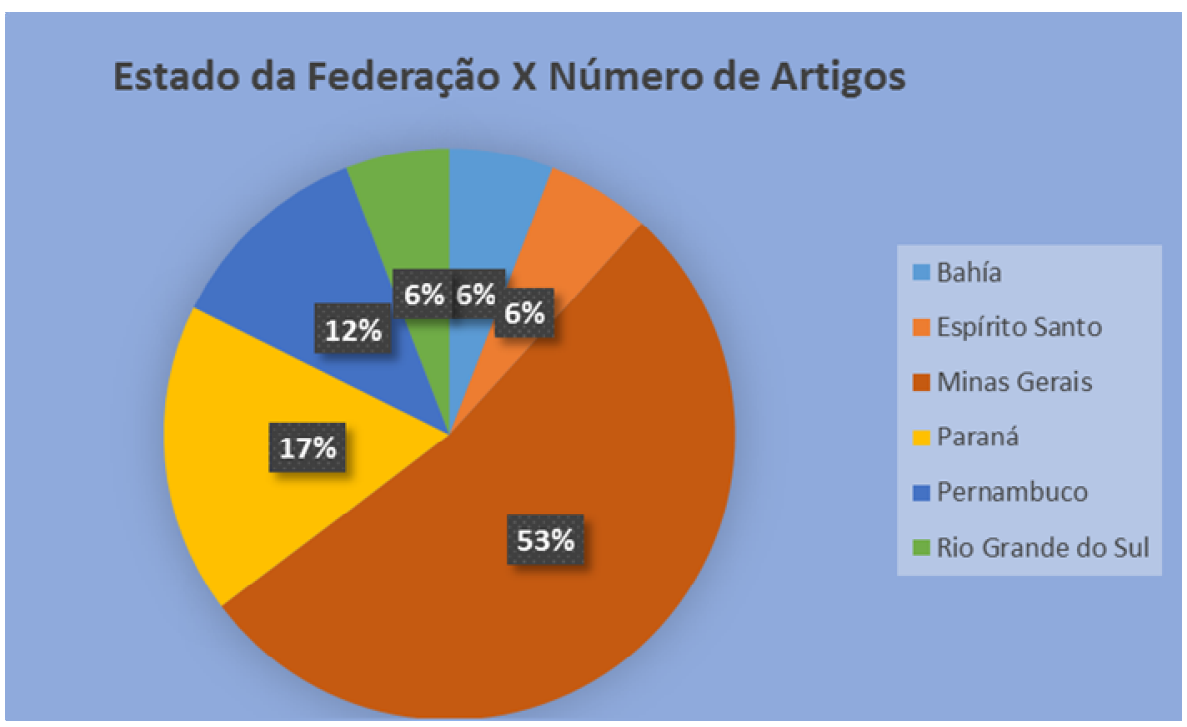

Observando-se ainda o Gráfico 2 pode-se notar que os artigos dos Minas Gerais, $50 \%$ são de Belo Horizonte e os demais são de Alfenas, Betim, Lavras, Ouro Branco e Santa Luzia.
Nossos agradecimentos aos pesquisadores que contribuíram com artigos, aos conselhos Científico e Editorial e aos Pareceristas ad hoc, cujo trabalho de avaliação permitiram a publicação desta edição. 